\title{
Detailed genomic characterization identifies high heterogeneity and histotype-specific genomic profiles in adrenocortical carcinomas
}

\author{
Simona Vatrano ${ }^{1} \cdot$ Marco Volante $^{1} \cdot$ Eleonora Duregon ${ }^{1} \cdot$ Jessica Giorcelli $^{1} \cdot$ Stefania Izzo $^{1} \cdot$ Ida Rapa $^{1}$. \\ Arianna Votta ${ }^{1}$ - Antonina Germano ${ }^{1}$. Giorgio Scagliotti ${ }^{1} \cdot$ Alfredo Berruti $^{2} \cdot$ Massimo Terzolo $^{3}$ - Mauro Papotti ${ }^{4}$
}

Received: 21 December 2017 / Revised: 30 January 2018 / Accepted: 31 January 2018 / Published online: 26 March 2018

(c) United States \& Canadian Academy of Pathology 2018

\begin{abstract}
Molecular characterization of adrenocortical carcinoma has been recently established, but the correlation between molecular profiles and clinical and pathological characteristics is still poorly defined with no data available about genetic heterogeneity along disease progression. In this scenario, a detailed molecular profile was correlated with clinical and pathological characteristics in adrenocortical carcinoma patients to identify potentially novel biomarkers. Targeted next-generation sequencing and copy number variation analyses for 18 most frequently altered genes in adrenocortical carcinoma were assessed on 62 adult cases (including 10 with matched primary and metastatic/recurrence samples) and results correlated with major clinical and pathological characteristics of tumors. A total of 433 somatic deleterious genetic alterations (328 gene mutations and 105 copy number variations) were identified in 57/62 cases, five resulted wild type for all genes tested. TERT, $C D K 4, Z N R F 3$, and $R B 1$ were altered in more than $30 \%$ of cases. Among histological variants genotypes were significantly different. Lowest mutation burden was found in the oncocytic type $(p=0.006)$, whereas the highest with a prevalence of $R B 1(p=0.001)$ and $C D K 4(p=0.002)$ was found in the conventional and myxoid ones, respectively. None of the 10 cases with matched samples showed a stable genotype along tumor progression, although allelic frequencies or percentages of altered nuclei at fluorescence in situ hybridization were in most cases similar among different tumor samples for genes that were stable along tumor progression. Among individual genes, an altered p53/Rb1 pathway was the strongest adverse molecular signature, being associated with high Ki-67 index, high tumor stage, aggressive disease status, and shorter disease-free survival. The genomic signature in adrenocortical carcinoma is changing along tumor progression and is associated with specific clinical and pathological features, including histological variant and prognosis.
\end{abstract}

Electronic supplementary material The online version of this article (https://doi.org/10.1038/s41379-018-0042-6) contains supplementary material, which is available to authorized users.

Marco Volante

marco.volante@unito.it

1 Department of Oncology, University of Turin at San Luigi Hospital, Orbassano, Turin, Italy

2 Oncology Unit, University of Brescia, Brescia, Italy

3 Internal Medicine, Department of Clinical and Biological Sciences, University of Turin at San Luigi Hospital, Orbassano, Turin, Italy

4 Department of Oncology, University of Turin at Molinette Hospital, Turin, Italy

\section{Introduction}

Despite better definition of the standard of care, adrenocortical carcinoma is a rare malignancy that has still a dismal prognosis, with a $20-40 \%$ 5-year survival. Adrenocortical carcinoma is not a uniform entity but is characterized by remarkable morphological (with several histological variants identified), clinical (with variable and poorly predictable outcome), and genetic heterogeneity. Pediatric adrenocortical carcinomas are frequently associated to Beckwith-Wiedemann syndrome (alterations of the insulin-like growth factor/IGF2) [1] and to Li-Fraumeni syndrome (TP53 mutations). TP53 germline mutations (p.R337H) are associated to pediatric adrenocortical carcinoma in Southern Brazil [2]. Recently, it has been recognized that in the adult population adrenocortical carcinoma can be associated with the Lynch syndrome [3]. Few driving somatic variants have been identified so far, and most of the available molecular data on adrenocortical carcinomas 
are restricted to gene expression, array-CGH, or methylome profiles assessed to compare adrenocortical carcinoma with the benign counterpart of adrenocortical adenoma. Mutations in TP53 gene are detectable as somatic events in about one-third of adult cases [2]. Mutations in CTNNB1 gene, coding for betacatenin protein, are detectable in a subset of adrenocortical carcinoma (about $40 \%$ ) but also in a similar percentage of adrenocortical adenomas. The role of these genes in the pathogenesis and progression of adrenocortical carcinoma is still controversial [4]. Recently, highly prevalent mutations of ZNRF3 (Zinc and ring finger protein 3) gene have been reported in adrenocortical carcinoma [5]. However, none of the above mentioned genomic alterations demonstrated to be prognostic or predictive markers of response to treatment. Many other mutations have been reported by whole genome sequencing though at very low prevalence. This confirms the heterogeneity of the molecular background of adrenocortical carcinoma and underlines the need for adequate investigations to define biomarkers that could have a positive impact in the clinical setting.

On the basis of these findings, we performed a detailed genomic characterization on 62 adrenocortical carcinoma cases and correlated mutational profile results with clinical and pathological characteristics. Furthermore, in ten cases of matched primary and recurrent/metastatic tissues the molecular signatures were compared to evaluate molecular heterogeneity during progression.

\section{Material and methods}

\section{Tissues samples}

Tumor samples from adrenocortical carcinoma patients undergoing surgery with curative intent were collected from the pathology files at the University of Torino, San Luigi Hospital (Orbassano, Turin, Italy). A total of 62 were selected based on enough leftover tissue from primary tumors for molecular analyses. Pathological re-classification included the recognition of major histological variants, namely the oncocytic, myxoid, and sarcomatoid as coded by the current WHO classification [6], applying the pathological criteria described in the available literature [7-11]. To define malignancy, the Weiss score for conventional and myxoid and adrenocortical carcinoma the Lin-Weiss-Bisceglia system for pure oncocytic ones were applied [7, 12]. Furthermore, the Helsinki Score was evaluated, as it has been recently indicated as a relevant prognostic system in adrenocortical carcinoma [13, 14]. Main clinical and pathological data (including age, gender, European Network for the Study of Adrenal Tumors-ENSAT_-stage, all parameters included in the Weiss score-WS, Helsinki score, hormonal secretion, and $\mathrm{Ki}-67$ proliferation index) were also recorded. Clinical data concerning outcome were available for all but one case. In ten cases, primary tumors were matched with corresponding metachronous recurrent or metastatic (liver or lung) lesions (for a total of 85 tissue samples overall enrolled in the study). All samples were de-identified, and cases anonymized by a pathology staff member not involved in the study. The study received ethical approval from the local review board of our Institution (protocol 20840 dated 22nd December 2016).

\section{DNA isolation and mutational analysis}

Genomic DNA was obtained from formalin-fixed paraffinembedded tissues after manual microdissection, for neoplastic cell enrichment (at least $50 \%$ of tumor cells), as previously reported [15]. Targeted next-generation sequencing analyses were performed in all samples using the Ion Torrent Personal Genome Machine (PGM, ThermoFisher Scientific), and an AmpliSeq custom panel targeting the entire coding sequence of 15 genes previously reported as adrenocortical carcinoma related: TP53, MSH2, MSH6, MLH1, PMS2, ATRX, DAXX, CTNNB1, MED12, MEN1, CTNNB1, PTCH1, APC, PTEN, ZNRF3. Targeted primers were designed using the AmpliSeq designer software v4.0 (ThermoFisher Scientific), see Supplementary Table S1. Twenty-five nanograms of genomic DNA were used for each multiplex PCR amplification. Library quality was evaluated by Agilent 2100 Bioanalyzer on-chip electrophoresis (Agilent Technologies) and libraries passing this step went in emulsion PCR performed with the OneTouch2 system (ThermoFisher Scientific). The libraries were loaded on a Ion 318chipv2 and then sequenced on the Ion PGM (ThermoFisher Scientific). Data analysis, including alignment to the reference genome and variant calling, was done using the Torrent Suite Software v.5.0 (ThermoFisher Scientific).

\section{Bioinformatics pipeline and experimental validation}

All genome coordinates are human genome build GRCh37/ hg19 and RefSeq sequences used for gene annotations are detailed in Supplemental Table 1. Genetic variants were annotated and filtered using a custom pipeline as previously reported [16]. To minimize the risk of calling alterations derived from sequencing errors or polymerase chain reaction (PCR)-based enrichment artifacts, all reads with a quality score $<30$ were excluded from further analyses. The discrimination of likely germline or likely somatic variants was performed on the basis of the following criteria: (i) known polymorphisms recorded in the public polymorphisms databases were removed from the analysis [17]; (ii) a variant read frequency range of $5-50 \%$ for all candidate alterations different from well-characterized somatic known hotspots [18, 19]. The following databases were used to 
(a)

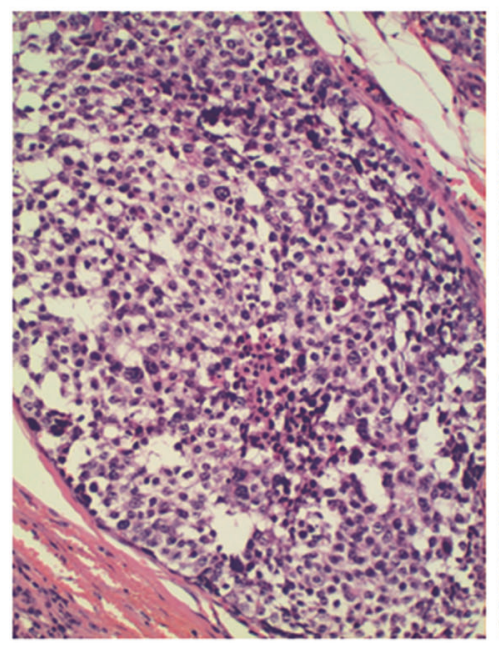

(b)

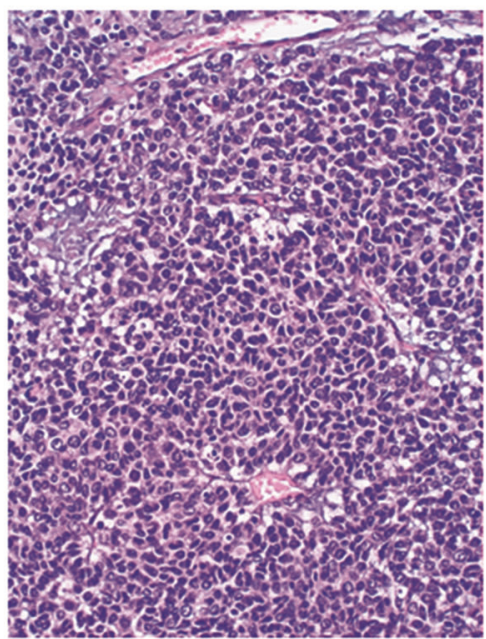

(c)

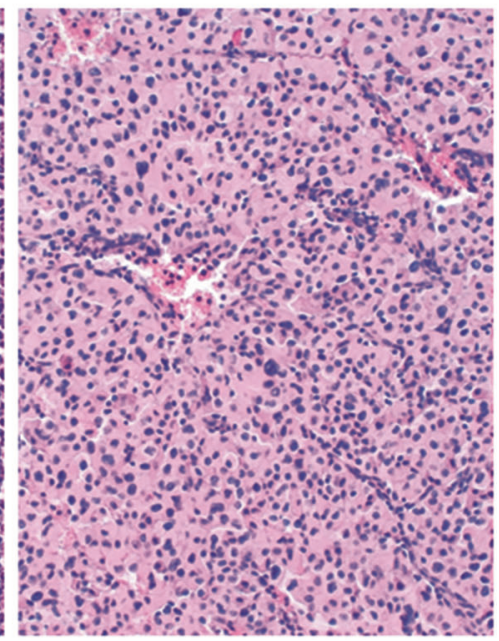

Fig. 1 The adrenocortical carcinoma morphologic variants identified in our cohort of patients. Architectural patterns in a conventional, b myxoid, and $\mathbf{c}$ oncocytic cases (hematoxylin and eosin staining, magnification $\times 20$ )

annotate the genetic alterations: COSMIC (version 70), Single Nucleotide Polymorphism (dbSNPs, build 137), 1000 Genomes Project, 6500 exomes of the National Heart, Lung and Blood Institute Exome Sequencing Project, and Non-synonymous Single Nucleotide Polymorphisms' Functional Predictions (dbNSFP, light version 1.3). In silico analyses were carried out to predict both the amino acid changes potentially deleterious to protein function and the clinical implications of genetic alterations not previously identified by means of sorting intolerant from tolerant and polyphen-2 (polymorphism phenotyping) algorithms. In addition, alignments and sequences were visually verified with the Integrative Genomics Viewer (IGV) v2.3 to further confirm the presence of the identified mutations [20]. PathVisio and Caleydo pathway-based analysis tools were used to cluster genetic alterations and copy number variations, respectively, into pathway [21].

\section{DNA copy number analysis}

Fluorescence in situ hybridization analysis was performed on $5-\mu \mathrm{m}$ sections as previously reported [15]. Different probe sets were used based on genomic loci to analyze according to the manufacturer's instructions. Three to five areas on each slide were analyzed to count at least 100-150 tumor cell nuclei. For RB1/CEP13 FISH (Zytolight, Zytovision), all cases with absence of one or both RB1 orange signal/s, but intact $13 \mathrm{q} 12$ green signals in more than $22 \%$ of nuclei, were reported as heterozygous or homozygous deleted, respectively [22]. For CDKN2A/CEP9 FISH (Zytolight, Zytovision), the nuclear probe patterns were normal if two green and two red signals were seen, whereas abnormal if $2 \mathrm{Green} / 1 \mathrm{Red}$ as loss of heterozygosity ( $\mathrm{LOH}$ ),
$2 \mathrm{Green} / 0 \mathrm{Red}$ as homozygous deletion and $1 \mathrm{Green} / 1$ Red as monosomy of chromosome 9 were seen. The cut-off value applied was $18 \%$ of altered nuclear signal for both $9 p$ loss of heterozygosity and homozygous loss of $9 \mathrm{p}$ (2Green/ 1Red-2Green/0Red) [23]. For ZNRF3/CEP22 FISH (Empire genomics), given the absence of fluorescence in situ hybridization interpretation criteria of this genomic locus, we followed the international guidelines [24]. Cases with absence of one or both ZNRF3 orange signal/s, but intact CEP22 green signals in more than $25 \%$ of nuclei, were reported as heterozygous or homozygous deleted, respectively. Finally, for both CDK4/CEP12 and TERT/ 5q31 FISH (Zytolight, Zytovision), amplification was defined when the ratio of signal counts Locus/Centromere was $>2$, with a cut-off of $10 \%$ of positive nuclei.

\section{Statistical analyses}

The $\chi^{2}$ test, Fisher's exact test, and Mann-Whitney tests were used to analyze the association between mutations and clinical pathological variables or to evaluate concordance between different results, as appropriate. Disease-free survival (DFS) was defined as the time between diagnosis and the first failure (either recurrence/metastasis or death). Univariate analysis was performed with Kaplan-Meier curves and the significance was verified by the log-rank test. Multivariate analysis was performed using a Cox proportional hazards model. Overall survival analysis was not performed due to the limited number of events. All analyses were performed using GraphPad software (Graphpad Software Inc., La Jolla, CA) and SPSSsoftware (IBM corporation, Armonk, USA). A $p$ value lower than 0.05 was considered statistically significant in all analyses. 
Table 1 Main clinical and pathological characteristics of ACC patients

Features

Total number

62

Median age,

years (range)

Sex no. (\%)

Male

$22(35 \%)$

Female

$40(65 \%)$

ENSAT stage no.

(\%)

I-II

$31(58 \%)$

III-IV

Not known

$22(42 \%)$

Hormonal

hypersecretion

no. (\%)

Cortisol

producing

Nonfunctiona

Other

Not known

Histologic type

no. (\%)

Conventional

$38(62 \%)$

Myxoid

Oncocytic

Weiss score

distribution no.

(\%)

$<6$

$13(21 \%)$

$\geq 6$

$49(79 \%)$

Helsinki score

distribution no.

(\%)

$<19$

$16(26 \%)$

$\geq 19$

$46(74 \%)$

Ki-67 index ${ }^{\mathrm{a}}$ no.

(\%)

$\leq 22 \%$

$34(55 \%)$

$>22 \%$

$28(45 \%)$

ENSAT European Network for the Study of Adrenal Tumors

${ }^{a}$ The selected cut-off corresponds to median value obtained in the present series.

\section{Results}

\section{Study cohort}

Adrenocortical carcinoma samples included 38 conventional, 7 myxoid, and 17 oncocytic variants (Fig. 1). No case of the sarcomatoid variant was recognized. Twentytwo were males and 40 females, with a median age at diagnosis of 45 years (range 18-82). According to the ENSAT stage, 5 patients presented with stage I, 26 patients with stage II, 15 patients with stage III, and 7 patients with stage IV. After a mean follow up of 34 months, 18 died of disease (conventional $=13$, myxoid $=3$; oncocytic $=2$ ). Thirty cases were hormone producing, 22 of which being cortisol-hypersecreting (Table 1). No significant differences were found between adrenocortical carcinoma variants, with the exception of age distribution (lower in conventional histotype; $p=$ 0.02 ) and a more favorable outcome in the oncocytic histotype $(p=0.05)$ (Supplementary Table S2).

\section{Prevalence of somatic alterations by means of targeted next-generation sequencing and fluorescence in situ hybridization}

The entire genomic profile (328 gene alterations and 105 somatic copy number variants) clusterized according to adrenocortical carcinoma histotypes is illustrated in Fig. 2a. Taken together, the results of DNA copy number alterations (105 somatic copy number variations) and targeted nextgeneration sequencing (328 genetic variations) analyses showed a high prevalence of somatic alterations, with 57/62 $(92 \%)$ cases harboring at least one alteration among studied genes, whereas five cases (8\%), which incidentally were all oncocytic adrenocortical carcinomas, did not harbor any somatic aberration. Somatic variants were clustered into four main pathways, among which the $\beta$-catenin pathway harbored the highest number of alterations (40\%), followed by p53/Rb (28\%), mismatch repair (18\%), and chromatinremodeling (14\%) pathways. Mutually exclusive alterations were observed in 13 cases (Fig. 2b).

All 62 samples were informative for targeted nextgeneration sequencing analysis, with adequate libraries of 690 amplicons each. According to bioinformatic pipeline, after read quality filtering, mapping, and alignment to the reference genome (hg19), the median sequencing depth was $896 \times$ and a total of 986 genetic variants (median 14; range: 1-44) were identified, either in exonic (284 synonymous and 338 non-synonymous, respectively, both likely germinal and likely somatic alterations) or regulatory regions (364 both likely germinal and likely somatic alterations). The nucleotide change spectrum was characterized by a predominance of cytosine:guanine $\rightarrow$ thymine:adenine transitions, as previously reported [25] and without significant difference among individual genes. All genetic variants which do not lead to a protein change (i.e., synonymous and regulative variants) or that were categorized as likely germline, based on the presence in public polymorphism databases or of their allelic frequency in the sample, were excluded from further analysis. Thus, we focused on 328 non-synonymous genetic alterations 
(median 5; range: 1-22), more likely having a causative role in tumorigenesis (Supplementary Table S3), that included 265 missense and 44 nonsense variations, 6 frameshifts, and 6 in-frame InDels variations. Almost half of them (40\%) were already reported in official mutational databases. The mean number of genetic alterations was 8 for conventional adrenocortical carcinomas, 7 for myxoid, and 2 for oncocytic ones, respectively; $42 / 62(68 \%)$ cases showed at least one alterations among sequenced genes, whereas 20 cases (32\%) were wild type for somatic genetic variants in the targeted genes. One conventional case (ACC14) showed a hypermutated profile with high number (128) of inactivating somatic variants mainly in APC, MED12, and MSH6 genes, as previously reported $[25,26]$. This case was excluded from all prevalence and statistical analyses of mutation burden, because of the large number of somatic alterations with skewing effect.

Among single genes, MED12, CTNNB1, and TP53 were the most commonly mutated (Supplementary Figure S1a). MED12 alterations were mainly missense and truncating mutations; four of these alterations were identified in more than one case, p.T1681I-p.P1824L-p.T2171L (in two patients), p.Q2115del (in three patients). Even though never reported before, they affected C-terminal region of the protein, known to be important for physically interact with $\beta$-catenin. In silico analyses confirmed the pathogenic role of these variants. Furthermore, in six cases truncating mutations were detected with a premature stop codon

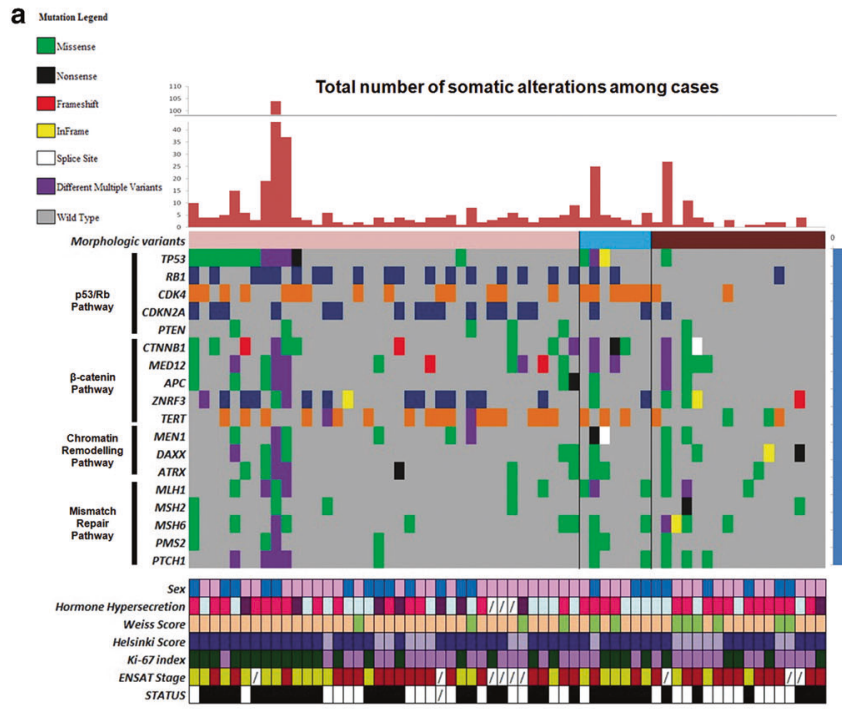

Fig. 2 Genomic signature in 62 adrenocortical carcinomas. a Heatmap describing distribution of the tumor-specific somatic alterations identified in each gene clustered according to specific signaling pathways (left). Histograms of the number of somatic alterations across samples (above) and genes (right), respectively, are reported, such as main clinical and pathological features (below). ENSAT European Network

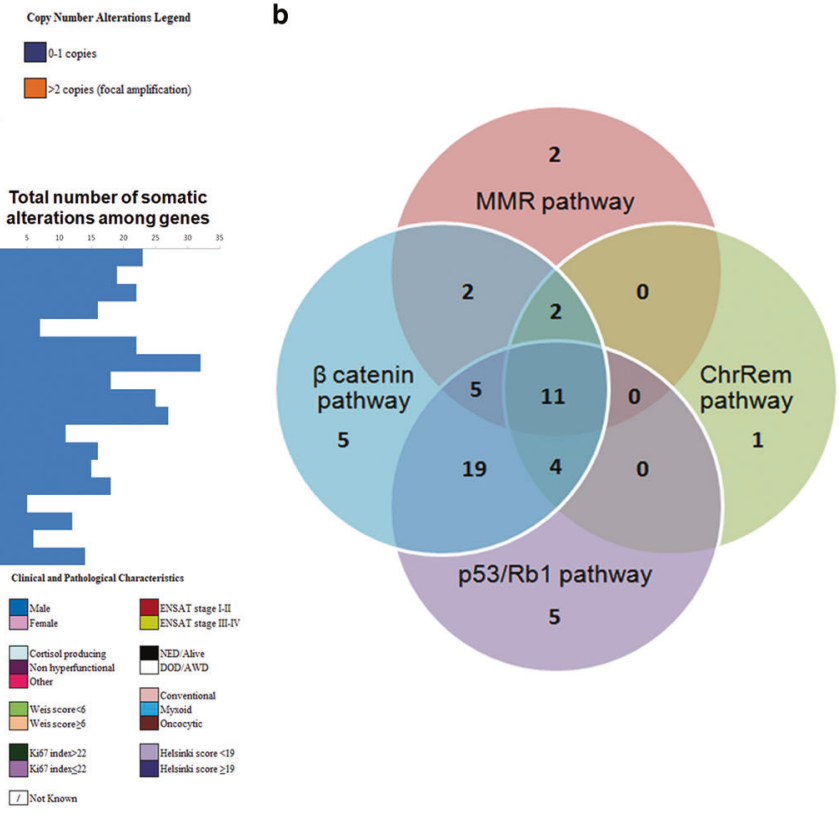

for the Study of Adrenal Tumors, Ned no evidence of disease, Dod dead of disease, Awd alive with disease. b Venn diagram illustrating the distribution of somatic alterations among the molecular pathways analyzed in our patient cohort. Chr Rem chromatin remodeling, $\mathrm{Rb}$ retinoblastoma, MMR MisMatch Repair

Table 2 Summary of copy number variations identified in adrenocortical carcinoma histological variants

\begin{tabular}{|c|c|c|c|c|c|c|c|}
\hline \multicolumn{2}{|c|}{ Genes (locus) analyzed } & \multicolumn{2}{|c|}{ Conventional $(n=38)$} & \multicolumn{2}{|c|}{ Myxoid $(n=7)$} & \multicolumn{2}{|c|}{ Oncocytic $(n=17)$} \\
\hline & & Loss & Gain & Loss & Gain & Loss & Gain \\
\hline $\mathrm{RB} 1 / 13 \mathrm{q} 12$ & $(13 q 14.2)$ & $18(47 \%)$ & l & $2(28 \%)$ & l & $1(6 \%)$ & l \\
\hline CDKN2A/CEN9 & $(9 \mathrm{p} 21.3)$ & $15(39 \%)$ & l & $2(28 / \%)$ & / & $1(6 \%)$ & l \\
\hline ZNRF3/22q13 & $(22 q 12.1)$ & $13(34 \%)$ & l & $1(14 \%)$ & l & I & l \\
\hline TERT/5q31 & $(5 \mathrm{p} 15.33)$ & l & $16(42 \%)$ & l & $3(43 \%)$ & I & $4(23 \%)$ \\
\hline CDK4/CEN12 & $(12 q 14.1)$ & l & $14(37 \%)$ & I & $6(86 \%)$ & I & $2(12 \%)$ \\
\hline
\end{tabular}


expected to downregulate protein levels. TP53 mutations were different and mostly clustered into DNA-binding or oligomerization domains. All of them have been already reported as somatic pathogenic mutations in several cancers, especially the mutation p.S116F, which was identified in two cases. Immunohistochemical analysis for p53 was performed as a surrogate validation test of the TP53 mutational status, with significant correlation between the two tests (Mann-Whitney test $p=0.005$ ) (Supplementary Figure S1b). Mutations in CTNNB1 gene were mainly localized in exon 3. Among these, three (p.Q28*-p.E53K-p. $\mathrm{S} 45 \mathrm{P})$ were recurrent and identified in two cases, respectively. All other variants are detailed in Supplementary Table S3.

All fluorescence in situ hybridization cases were able to generate an informative genomic profile. Most cases (43/62, $69 \%)$ exhibited either gains of oncogenes (CDK4 and $T E R T)$ or loss of tumor suppressor genes (CDKN2A, RB1, and ZNRF3) (Supplementary Table S4, Table 2, Fig. 3). TERT and ZNRF3 genes, although showing also somatic variants, more frequently harbored copy number variations, indeed 23/62 (37\%) cases harboring amplification of TERT and 19/62 (30\%) cases deletions of ZNRF3. Loss of $C D K N 2 A$ and $R B 1$ loci was detected in 18/62 (\%) and 21/62 (\%) cases, respectively; whereas amplifications of $C D K 4$ locus were identified in 22/62 (35\%) patients. Moreover, $13 / 62(21 \%)$ cases had polysomy of CDK4 and/or TERT loci, although these were not considered altered for statistical purposes due to their equivocal functional and pathogenic significance.

\section{Clinical and pathological correlation with integrated genomic profile}

Correlation analysis between molecular data and clinical pathological variables was performed and summarized in Table 3. Of note, we observed higher prevalence of $R B 1$ $(p=0.01), C D K 4 \quad(p=0.003)$, and CDKN2A $(p=0.04)$ alterations in the conventional and myxoid histotypes; TP53 mutations were associated with a higher Ki-67 index, higher Helsinki score, higher tumor stage, and aggressive disease status, either as single gene mutation (all $p<0.04$ ) or as mutational burden of the $\mathrm{p} 53 / \mathrm{Rb}$ pathway (all $p<0.04$ ); tumors with TERT and CTNNB1 alterations were associated with higher Weiss score $(p=0.03)$ and higher Ki-67 index $(p=0.04)$, respectively. Furthermore, $P M S 2$ and PTCH1 genetic alterations were more prevalent in male compared with female patients (both $p<0.001$ ), whereas CDK4 alterations were associated with higher Helsinki score $(p=0.03)$. Interestingly, the overall mutational burden, as well as the mutational burden of $\beta$-catenin and $\mathrm{p} 53 / \mathrm{Rb}$ pathways, were significantly different among histological variants, with the lowest rate in the oncocytic one ( $p=0.006, p=0.02$ and $p=0.0002$, respectively) (Fig. 4).
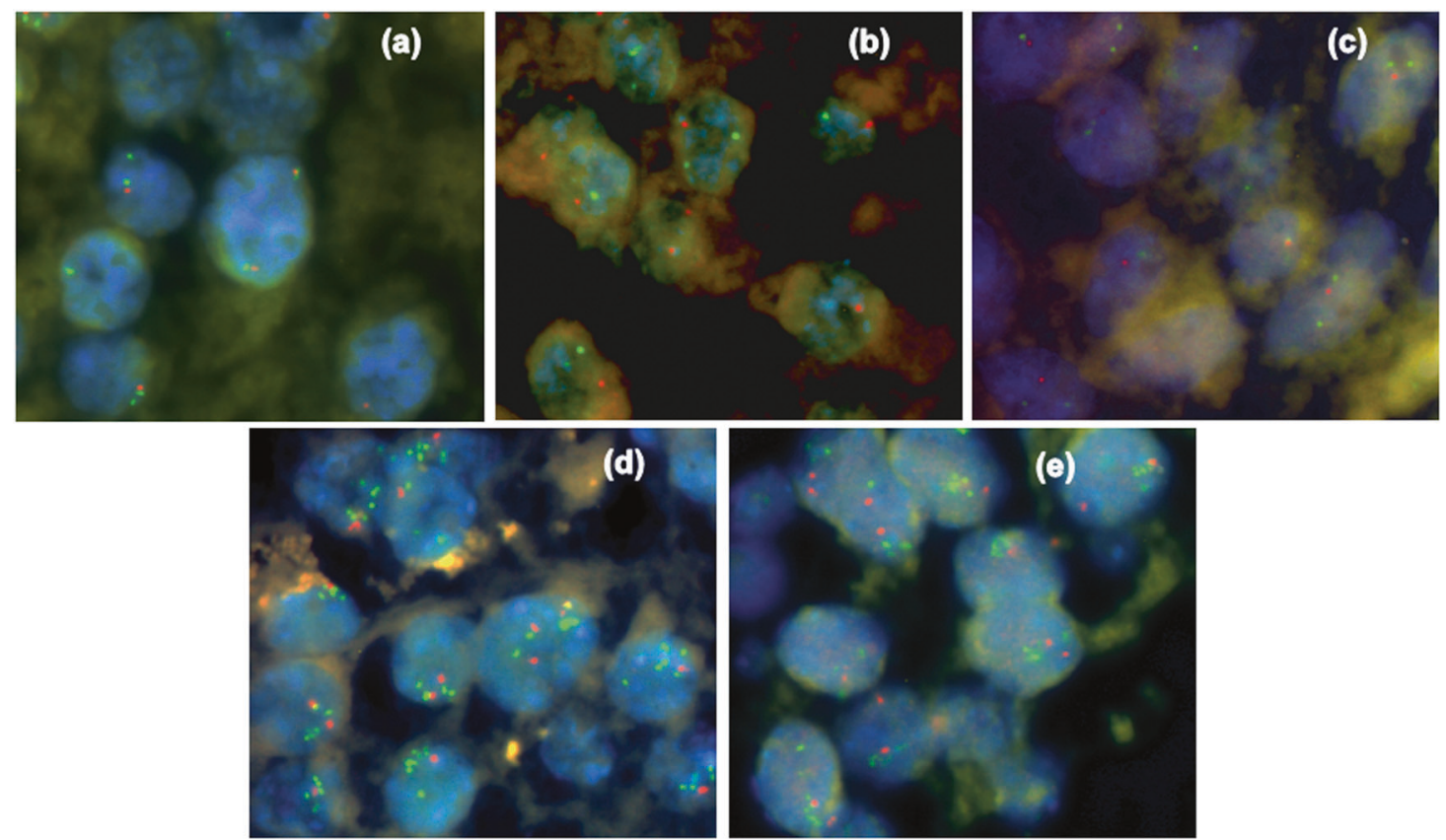

Fig. 3 Fluorescence In Situ Hybridization of gene loci analyzed our cohort of patients. Images of different chromosomal loci investigated in our cases and different alterated patterns identified: a $R B 1$ (conventional adrenocortical carcinoma), b CDKN2A (conventional

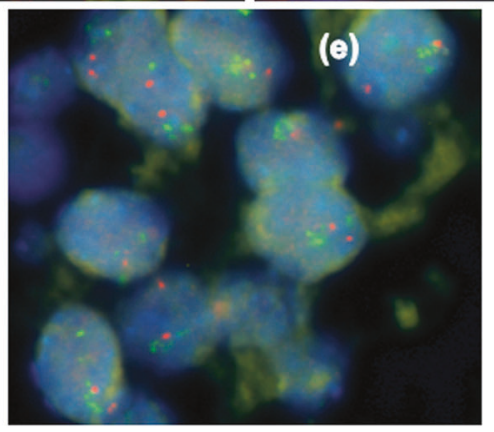

adrenocortical carcinoma), c ZNRF3 (conventional adrenocortical carcinoma), $\mathbf{d} C D K 4$ and (myxoid adrenocortical carcinoma), and e TERT (myxoid adrenocortical carcinoma) (magnification $\times 100$ ) 






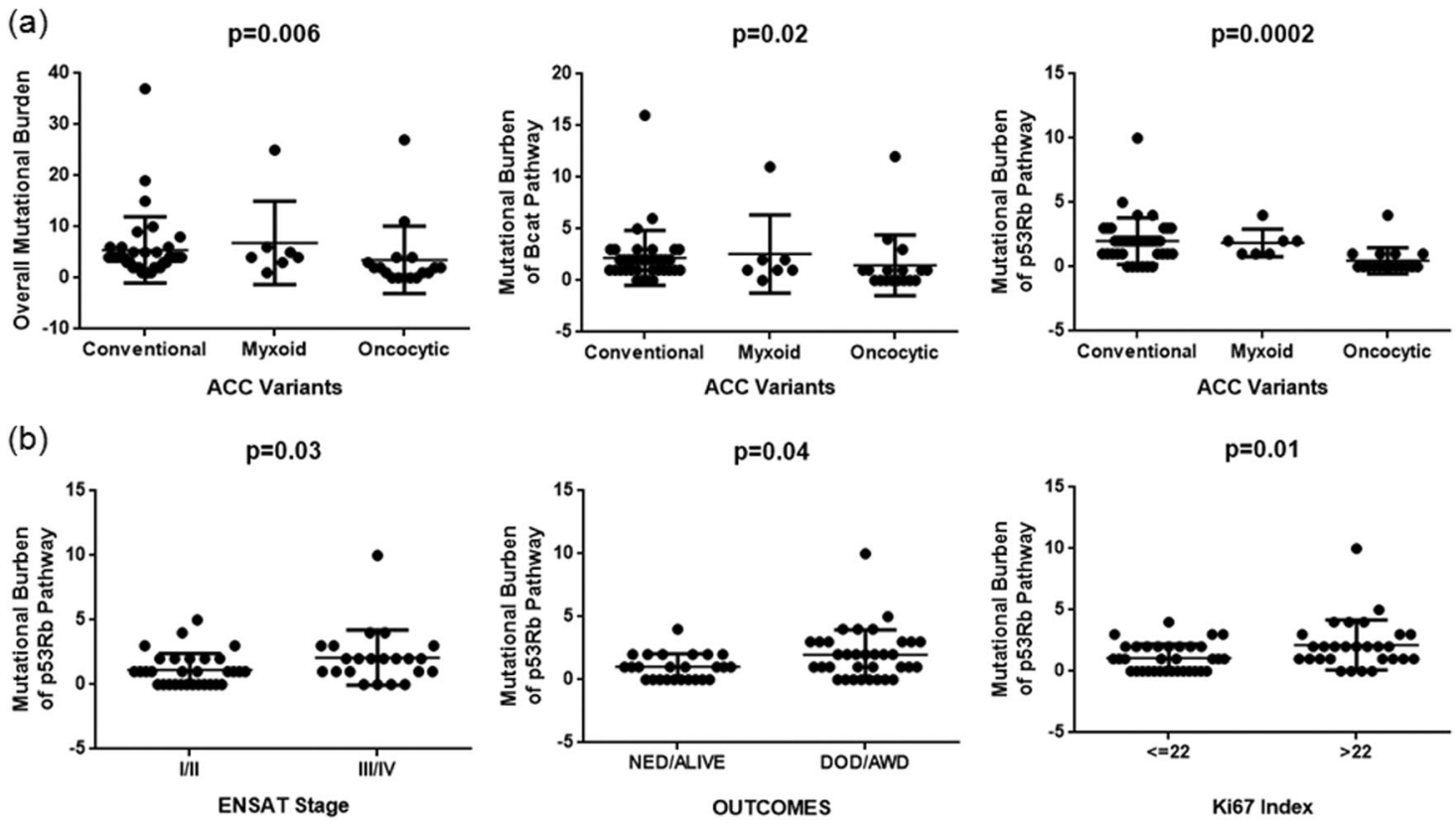

Fig. 4 Correlation between mutational burden and the clinical and pathological features in 62 adrenocortical carcinomas. a Overall mutational burden, mutational burden of $\beta$-catenin, and $\mathrm{p} 53 / \mathrm{Rb}$

pathways in adrenocortical carcinoma variants; $\mathbf{b}$ mutational burden of p53/Rb pathway as compared to selected clinical and pathological variables

\section{Survival analysis}

Median DFS was of 22.5 months. At univariate analysis, a conventional/myxoid histotype $(p=0.01)$, a high Weiss score $(p=0.01)$, a high Helsinki score $(p=0.002)$, a high mitotic index $(\mathrm{p}=0.004)$, and high $\mathrm{Ki}-67$ index $(p=$ 0.0004) were all predictive of poor DFS (Fig. 5); as well as harboring an high mutational burden of $\beta$-catenin $(p=$ $0.04), \mathrm{p} 53 / \mathrm{Rb}(p=0.02)$, and chromatin-remodeling $(p=$ 0.03 ) pathways, using the median number of somatic alterations in each pathway as the arbitrary cut-off value (Fig. 5, Table 4). In multivariate analysis, Ki-67 and Helsinki score remained independent predictors of DFS (hazard ratio $(\mathrm{HR})=2.60,95 \%$ confidence interval $(\mathrm{CI})$ : 0.89-7.64, $p=0.04$; HR $=2.59,95 \%$ CI: 0.72-9.13, $p=0.01$ ), together with an high mutational burden of mismatch repair pathway $(\mathrm{HR}=0.16,95 \% \mathrm{CI}: 0.03-0.73, p=0.01)$.

\section{Re-assessment of the genomic profiling at the time of disease progression}

The genomic profile obtained from matched samples is illustrated in Fig. 6 (Supplementary Table S5). Overall, a very high degree of genetic heterogeneity was observed. None of the patients showed a stable genotype between diagnosis and tumor progression and both acquisition and loss of somatic genomic alterations were observed. The number of molecular events did not allow a statistical comparison and in most instances the heterogeneity was linked to molecular alterations encountered at low allelic frequency or percentage of nuclei with copy number variations. However, arbitrarily considering only cases with a change in the percentage of allelic frequency/nuclei with copy number variations above $50 \%$, the events acquired during progression were more frequently gene mutations (13/14), acquired rather than lost as compared to the primary tumor (13/14) and involving a relative small set of genes, mostly (4/13) belonging to the mismatch repair pathway. Indeed, allelic frequencies or percentages of altered nuclei at fluorescence in situ hybridization were in most cases similar among different tumor samples for genes that were stable along tumor progression (Fig. 7).

\section{Discussion}

The genomic characterization of adrenocortical carcinoma has been recently assessed by high-throughput technologies approaches, leading to the identification of specific molecular profiles that allow adrenocortical carcinoma stratification in molecular subtypes with distinct clinical outcome [5, 25-27]. This is the first study focusing on the most prevalent molecular alterations previously identified in adrenocortical carcinoma with the aim to investigate their correlation with pathological and clinical characteristics and to assess genetic heterogeneity along the process of tumor progression. The use of molecular markers for diagnostic 
ACC Variants

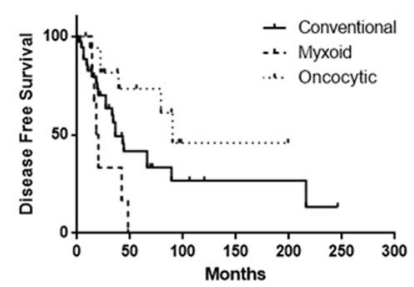

Helsinki Score
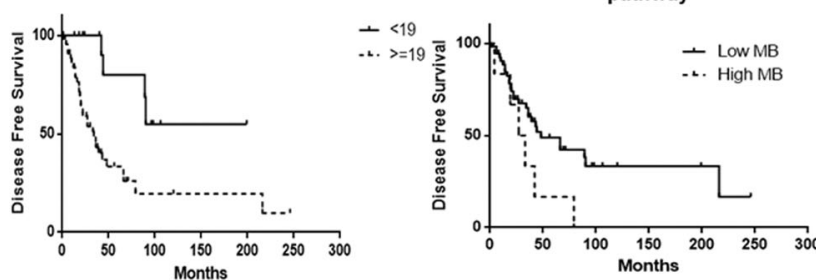

Fig. 5 Kaplan-Meier survival curves of disease-free survival (DFS). Patients segregated according to adrenocortical carcinoma variants $(p$ $=0.01)$, Weiss score $(p=0.01)$, mitotic index $(p=0.004)$, Ki-67 index $(p=0.0004)$, Helsinki score $(p=0.002)$, and to the median
Mitotic Index

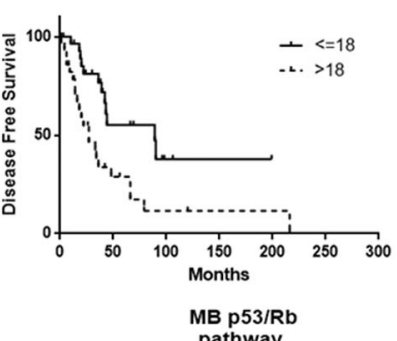

pathway

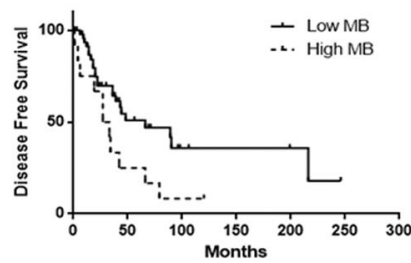

number of somatic alterations in $\beta$-catenin $(p=0.04), \mathrm{p} 53 / \mathrm{Rb}(p=$ $0.02)$ and chromatin-remodeling $(p=0.03)$ pathways. MB mutational burden, Chr Rem chromatin remodeling, $\mathrm{Rb}$ retinoblastome

Table 4 Survival analysis of clinic pathological and genomic variables

\begin{tabular}{|c|c|c|c|c|}
\hline \multirow[t]{2}{*}{ Parameters } & \multicolumn{2}{|c|}{$\begin{array}{l}\text { Univariate analysis of disease-free } \\
\text { survival }\end{array}$} & \multicolumn{2}{|c|}{$\begin{array}{l}\text { Multivariate analysis of disease-free } \\
\text { survival }\end{array}$} \\
\hline & HR (CI 95\%) & $p$ value & HR (CI 95\%) & $p$ value \\
\hline Sex (M vs. F) & $0.87(0.43-1.73)$ & 0.69 & $1.02(0.33-3.16)$ & 0.8 \\
\hline Age (above vs. below median) & $1.25(0.64-2.47)$ & 0.4 & $1.98(0.75-5.28)$ & 0.2 \\
\hline Histotype (conventional /myxoid vs. oncocytic) & $2.49(1.08-4.46)$ & 0.011 & $0.49(0.17-1.44)$ & 0.2 \\
\hline Mitotic index ${ }^{\mathrm{a}}(>18$ vs. $\leq 18)$ & $2.83(1.39-5.76)$ & 0.004 & $2.13(0.22-2.08)$ & 0.06 \\
\hline Weiss score ( $\geq 6$ vs. $<6$ ) & $2.41(1.18-5.26)$ & 0.01 & $1.07(0.31-3.68)$ & 0.9 \\
\hline Helsinki score ( $\geq 19$ vs. $<19)$ & $3.10(1.49-6.44)$ & 0.002 & $2.59(0.72-9.13)$ & 0.01 \\
\hline Ki-67 index ${ }^{\mathrm{a}}$ (>22 vs. $\left.\leq 22\right)$ & $3.76(1.80-7.83)$ & 0.0004 & $2.60(0.89-7.64)$ & 0.04 \\
\hline ENSAT stage (I/II vs. III/IV) & $0.58(0.26-1.17)$ & 0.13 & $1.50(0.52-4.33)$ & 0.4 \\
\hline $\begin{array}{l}\text { Overall mutational burden (above vs. below } \\
\text { median) }\end{array}$ & $0.59(0.30-1.21)$ & 0.16 & $0.85(0.25-2.89)$ & 0.1 \\
\hline $\mathrm{P} 53 / \mathrm{Rb}$ pathway $(\mathrm{MB}>2$ vs. $\mathrm{MB} \leq 2)$ & $2.90(1.18-7.13)$ & 0.02 & $2.18(0.48-9.78)$ & 0.3 \\
\hline Chr/Rem. pathway $(\mathrm{MB}>1$ vs. $\mathrm{MB} \leq 1)$ & $2.79(1.10-7.11)$ & 0.03 & $1.6(0.37-7.05)$ & 0.5 \\
\hline$\beta$ cat pathway $(\mathrm{MB}>3$ vs. $\mathrm{MB} \leq 3)$ & $3.61(1.04-12.48)$ & 0.04 & $1.71(0.26-11.95)$ & 0.5 \\
\hline MMR pathway (MB > 2 vs. $\mathrm{MB} \leq 2$ ) & $0.96(0.36-2.51)$ & 0.9 & $0.19(0.03-0.73)$ & 0.01 \\
\hline
\end{tabular}

ENSAT European Network for the Study of Adrenal Tumors, $H R$ hazard ratio, CI confidence interval, Chr. Rem chromatin remodeling, $\beta c a t \beta-$ catenin, $M M R$ mismatch repair, $M$ male, $F$ female, vs. versus, $M B$ mutational burden

a The selected cut-off corresponds to median value obtained in the present series

purposes was not an aim of the present study and for this reason benign adrenocortical tumors were not included.

Our analysis, performed on 62 sporadic adult adrenocortical carcinomas at the genetic and genomic level by targeted next-generation sequencing and fluorescence in situ hybridization techniques, revealed a very high mutational burden in the studied cohort of patients, with $92 \%$ of the samples having at least one somatic alteration in the evaluated genes. Although the genetic assessment in our study was targeted to a group of 18 genes and not to the whole genome, this prevalence of mutations is higher than others previously reported [25-27]. This might be partially explained by specific pre-analytical and analytical workflows of our targeted next-generation sequencing molecular analysis aimed to identify somatic alterations $[16,28]$. The greater depth of sequencing (more than 500x) performed by 


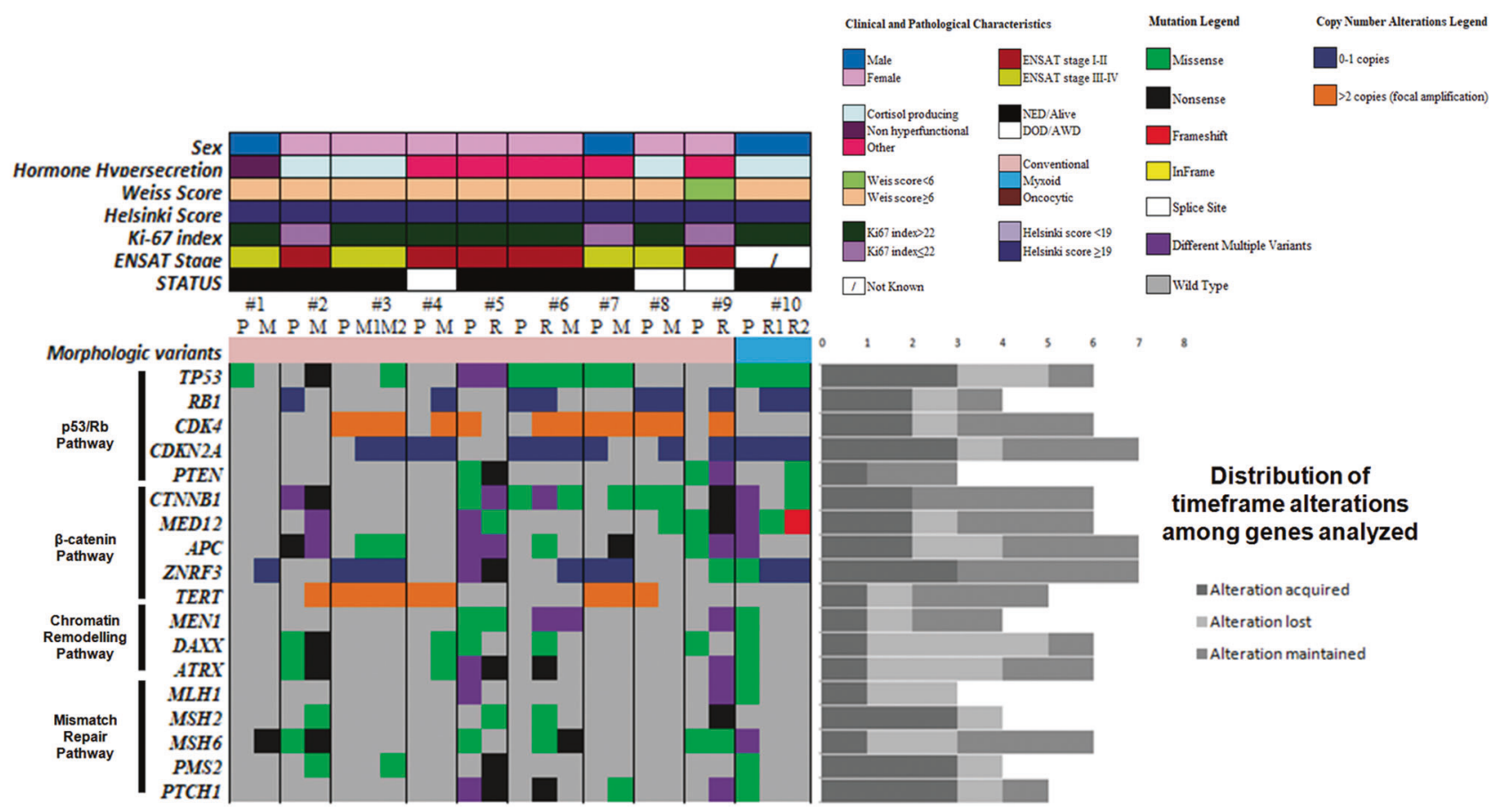

Fig. 6 Overview of somatic alterations identified for matched primary and recurrent/metastatic tissues in 10 adrenocortical carcinoma samples. Distribution of tumor-specific somatic variants and copy number changes are illustrated in the heatmap showing for each sample the

us could explain the differences between the studies and might improve detection of subclonal events. This is particularly relevant when the specimens show a low tumor purity and high heterogeneity as adrenocortical carcinoma, which calls into question the suitability of whole-exome sequencing as the platform of choice with diagnostic and clinical purposes. Moreover, it is worth of consideration that in the vast majority of cases, molecular alterations in the four main pathways covered by our analysis were not mutually exclusive and that we selected these genes based on results obtained by most wide genomic analysis. This highlights how an additive rather than single pathway deregulation mechanisms is the hallmark of adrenocortical carcinoma.

\section{Molecular characterization of adrenocortical carcinoma variants}

The mutational burden was different among adrenocortical carcinoma histological variants, showing a higher prevalence of somatic alterations in conventional and myxoid variants as compared to the oncocytic one $(p=0.006)$. Indeed, a small proportion of patients (8\%) did not harbor any somatic alterations in the analyzed genes, and, interestingly, they were all oncocytic. These data might partly be associated with the previously described more indolent clinical behavior of oncocytic adrenocortical carcinoma $[8$, 29]. In fact, also in the present series, this histotype was corresponding recurrence/metastasis. Left: genes are clustered according to specific signaling pathways; right: histograms of timeframe alterations among genes. Main clinical and pathological features are detailed above

associated with a more favorable prognosis at univariate analysis for DFS, as compared to conventional and myxoid tumor types $(\mathrm{HR}=2.49,95 \% \mathrm{CI}: 1.08-4.46, p=0.01)$. However, among the $8 \%$ of cases with no detectable molecular alterations, 3 out of 5 had a Weiss score $>6$ and 3 out of 5 only had a indolent clinical behavior. Thus, at least in the small number of cases available, there were no specific clinical or pathological characteristics in these tumors, apart from being oncocytic. The favorable prognosis of oncocytic adrenocortical carcinoma is furthermore highlighted in the present study by the identification of a significant correlation with a lower prevalence of mutations in the Wnt/ $\beta$-catenin $(p=0.02)$ and $\mathrm{p} 53 / \mathrm{Rb} \quad(p=0.0002)$ pathways, both known to be individually associated to a more aggressive adrenocortical carcinoma phenotype. This finding was also confirmed in studies of patients harboring these alterations associated with poor DFS either as individually mutated genes or as altered pathways (see below). On the other hand, the more frequent inactivation of $R B 1$ $C D K N 2 A$ and gain of function alterations of $C D K 4$, crucial in G1-S phase cell-cycle progression, in myxoid and conventional adrenocortical carcinomas are in line with the more aggressive and proliferative phenotype of these tumors. This is also confirmed by the association of $C D K 4$ $(p=0.03)$ alterations with higher Helsinki score, which has been recently identified as a valuable system to predict prognosis in morphological variants of adrenocortical 


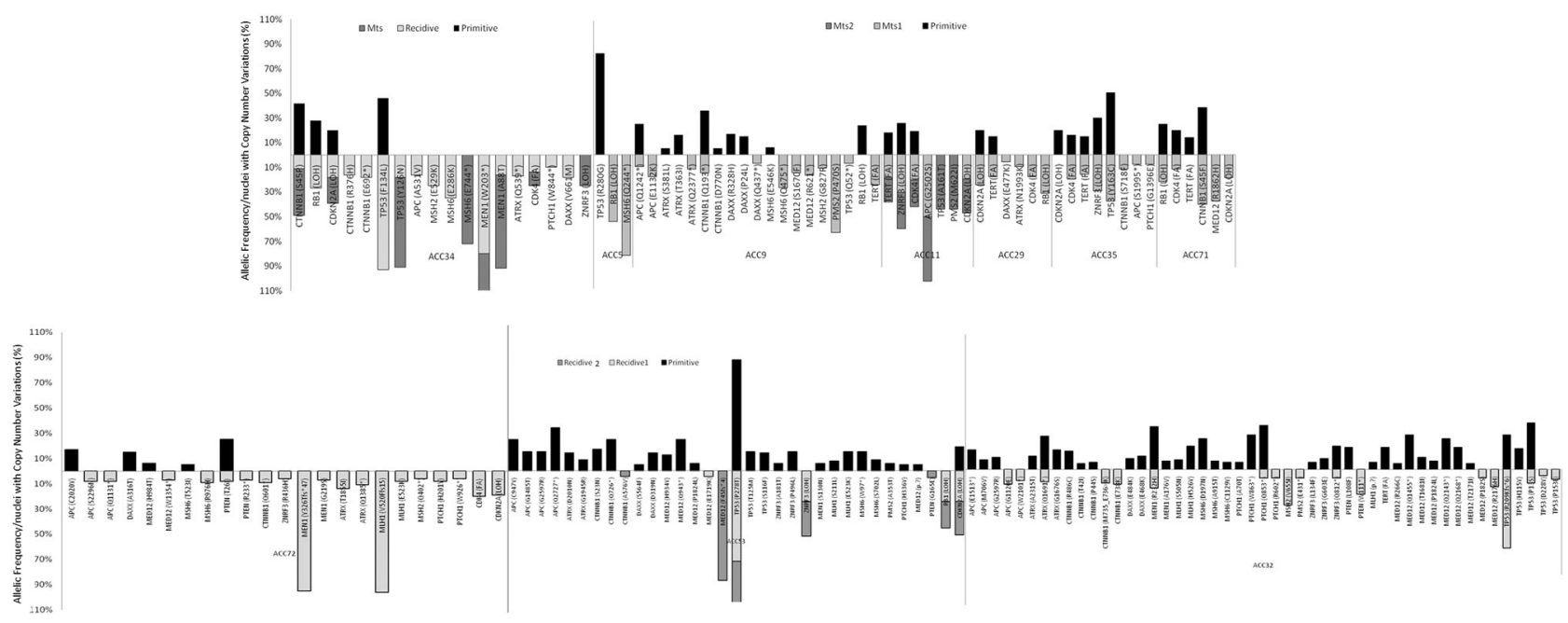

Fig. 7 Illustartion and distribution of somatic alterations and their frequencies identified along tumor progression. Somatic alterations and their allelic frequencies or percentage of nuclei with copy number

carcinoma, outperforming the currently established prognostic parameters $[13,14]$. Drugs targeting the cell-cycle pathway, as the CDK4 inhibitor (PD0332991), currently tested in phase I clinical trials (www.clinicaltrials.gov), may represent a new therapeutic strategy for patients with advanced adrenocortical carcinomas.

\section{Correlation of molecular profile with other clinical and pathological features}

Among the different molecular alterations associated with specific clinical and pathological parameters, TP53 and CTNNB1 alone were the most altered (>20\%), with somatic variations already reported and cancer associated in somatic genomic databases. These genes are well known to have a role in adrenocortical carcinoma pathogenesis [26, 27], as early (CTNNB1) or late (TP53) events in the oncogenesis. Among TP53 variants identified in our cohort, none of our patients harbored the germline mutation p. $\mathrm{R} 337 \mathrm{H}$, whereas we detected either several TP53 mutations with a known dominant negative function or less common mutations associated with absence of protein expression. This finding could explain the good concordance between p53 immunoreactivity and the TP53 genetic status. TP53 mutations were significantly associated with high Ki-67 index, high Helsinki score, high tumor stage, and aggressive disease status (all $p<0.04$ ). These results confirm that TP53 inactivation should be a late event in adult adrenocortical carcinoma oncogenesis [30], and highly prevalent in sporadic adrenocortical carcinoma with a more advanced stage and decreased DFS [31-33]. Our data expand these observations to the concurrent analysis of several genes acting in the p53/ Rb1 pathway, reinforcing the association of a high mutational rate in $\mathrm{p} 53 / \mathrm{Rb}$ pathway genes with poor DFS. variation identified in 10 adrenocortical carcinoma samples with matched primary and recurrent/metastatic. ACC adrenocortical carcinoma, Mts metastasis

Similarly, CTNNB1 somatic alterations are associated with poor outcome in adrenocortical carcinoma [34]. In line with these results, in our study a high mutational rate of $\mathrm{Wnt} / \beta$-catenin pathway genes showed a poor DFS. The detected $C T N N B 1$ gene alterations were mainly localized in exon 3 and known to be pathogenic activating mutations with leading to stabilization of $\beta$-catenin. CTNNB1 alterations are also associated with a higher Ki-67 index $(p=$ 0.04). However, only approximately one quarter of adrenocortical carcinoma cases with $\beta$-catenin nuclear delocalization is explained by activating mutations of CTNNBI gene $[4,34]$. Interestingly, in our study, molecular alterations of TERT and MED12 were among the most frequent ones, as previously reported [25]. As they are involved in Wnt/ $\beta$-catenin pathway interacting physically with $\beta$ catenin [35], their alterations could influence the $\beta$-catenin protein activity in adrenocortical carcinoma.

We also identified an association with poor survival for the chromatin-remodeling pathway ( $\mathrm{HR}=0.19,95 \% \mathrm{CI}$ : $0.03-0.73, p=0.01$ ), that (together with $\mathrm{Wnt} / \beta$-catenin and $\mathrm{p} 53 / \mathrm{Rb}$ pathways) was previously found altered in a subset of unfavorable outcome patients [25], but not by other groups [26, 27]. The somatic alterations identified in $D A X X$, $A T R X$, and MEN1 genes were mainly loss of function and/ or affecting important functional domains of the corresponding proteins.

\section{Genomic heterogeneity along tumor progression}

The comparison of the genomic profiles from matched primary vs. recurrent/metastatic samples showed a high degree of genetic heterogeneity. None of the patients analyzed $(n=10)$ showed a stable genotype between diagnosis and the time of tumor progression. In fact, in all cases either 
gain or loss of somatic genomic alterations during disease progression were observed and no significant association was observed between genes and cases although-with all the limitations due to small sample size-an enrichment of mismatch repair pathway alterations was observed. On the other hand, for those genes whose alterations were maintained in the different tumor samples of individual patients, a relative stability of percentage of allelic frequency or tumor cell nuclei with copy number variation was observed. Even if molecular heterogeneity has been already described in other tumors, such as breast, lung, and colon cancer [36, 37], it has never been reported in adrenocortical carcinoma, so far. Absence of recurrent gains or losses of molecular alterations in matched samples, makes difficult to discriminate between those that are crucial for survival and proliferation during the metastatic process from passenger ones. These data, provide additional support to a branched evolution model during oncogenesis in adrenocortical carcinoma, as recently reported in other malignancies [38, 39]. Moreover, the high degree of heterogeneity would represent a relevant pitfall for adrenocortical carcinoma molecular testing in the clinical practice and should be considered in future studies aimed to identify prognostic or predictive markers.

In conclusion, in this study we demonstrated that: (a) the genomic signature in adrenocortical carcinoma differs among histological variants and is associated to specific clinical and pathological characteristics; (b) p53/Rb pathway alterations are the most relevant prognostic molecular markers in adrenocortical carcinoma; (c) the genomic signature in adrenocortical carcinoma is highly unstable along tumor progression, thus emphasizing the potential clinical problem of molecular biomarker testing in advanced adrenocortical carcinoma patients.

Acknowledgements This work was supported by grants from Associazione Italiana per la Ricerca sul Cancro (AIRC, Milan; No. IG/ $14820 / 2013$ to MP).

\section{Compliance with ethical standards}

Conflict of Interest The authors declare that they have no conflict of interest.

\section{References}

1. Papotti M, Duregon E, Volante M, McNicol AM. Pathology of the adrenal cortex: a reappraisal of the past 25 years focusing on adrenal cortical tumors. Endocr Pathol. 2014;25:35-48.

2. Giacomazzi J, Selistre SG, Rossi C, et al. Li-Fraumeni and Li-Fraumeni-like syndrome among children diagnosed with pediatric cancer in Southern Brazil. Cancer. 2013;119: 4341-9.

3. Raymond VM, Everett JN, Furtado LV, et al. Adrenocortical carcinoma is a lynch syndrome-associated cancer. J Clin Oncol. 2013;31:3012-8.
4. Gaujoux S, Grabar S, Fassnacht M, et al. $\beta$-catenin activation is associated with specific clinical and pathologic characteristics and a poor outcome in adrenocortical carcinoma. Clin Cancer Res. 2011;17:328-36.

5. Zheng S, Cherniack AD, Dewal N, et al. Comprehensive Pangenomic characterization of adrenocortical carcinoma. Cancer Cell. 2016;29:723-36.

6. Giordano TJ, Chrousos GP, De Krijger RR, Gill A, Kawashima A, Koch CA, Medeiros LJ, Merino MJ, Papathomas TG, Papotti M, Sasano HR, Weiss LM. Tumours of the adrenal cortex. In: Lloyd RV, Osamura RY, Kloppel G, Rosai J, editors. WHO classification of tumours of endocrine organs. 4th ed. Lyon: WHO; 2017. pp. $163-8$.

7. Bisceglia M, Ludovico O, Di Mattia A, et al. Adrenocortical oncocytic tumors: report of 10 cases and reviews of the literature. Int J Surg Pathol. 2004;12:231-43.

8. Duregon E, Volante M, Cappia S, et al. Oncocytic adrenocortical tumors: diagnostic algorithm and mitochondrial DNA profile in 27 cases. Am J Surg Pathol. 2011;35:1882-93.

9. Weissferdt A, Phan A, Suster S, Moran CA. Myxoid adrenocortical carcinoma: a clinicopathologic and immunohistochemical study of 7 cases, including 1 case with lipomatous metaplasia. Am J Clin Pathol. 2013;139:780-6.

10. Papotti M, Volante M, Duregon E, et al. Adrenocortical tumors with myxoid features: a distinct morphologic and phenotypical variant exhibiting malignant behavior. Am J Surg Pathol. 2010;34:973-83.

11. Papathomas TG, Duregon E, Korpershoek E, et al. Sarcomatoid adrenocortical carcinoma: a comprehensive pathological, immunohistochemical, and targeted next-generation sequencing analysis. Hum Pathol. 2016;58:113-22.

12. Lau SK, Weiss LM. The Weiss system for evaluating adrenocortical neoplasm: 25 years later. Hum Pathol. 2009;40:757-68.

13. Pennanen M, Heiskanen I, Sane T, et al. Helsinki score-a novel model for prediction of metastases in adrenocortical carcinomas. Hum Pathol. 2015;46:404-10.

14. Duregon E, Cappellesso R, Maffeis V, et al. Validation of the prognostic role of the "Helsinki Score" in 225 cases of adrenocortical carcinoma. Hum Pathol. 2017;62:1-7.

15. Vatrano S, Righi L, Vavalá T, et al. Molecular and histological changes in post-treatment biopsies of non-squamous non-small cell lung cancer: a retrospective study. Target Oncol. 2016;11: 157-66.

16. Righi L, Vatrano S, Di Nicolantonio F, et al. Retrospective multicenter study investigating the role of targeted next-generation sequencing of selected cancer genes in mucinous adenocarcinoma of the lung. J Thorac Oncol. 2016;11:504-15.

17. Kumar A, White TA, MacKenzie AP, et al. Exome sequencing identifies a spectrum of mutation frequencies in advanced and lethal prostate cancers. Proc Natl Acad Sci USA. 2011;108: 17087-92.

18. Bourgon R, Lu S, Yan Y, et al. High-throughput detection of clinically relevant mutations in archived tumor samples by multiplexed PCR and next-generation sequencing. Clin Cancer Res. 2014;20:2080-91.

19. Choudhary A, Mambo E, Sanford T, et al. Evaluation of an integrated clinical workflow for targeted next-generation sequencing of low-quality tumor DNA using a 51-gene enrichment panel. BMC Med Genom. 2014;7:62.

20. Robinson JT, Thorvaldsdottir H, Winckler W, et al. Integrative genomics viewer. Nat Biotechnol. 2011;29:24-26.

21. Cirillo E, Parnell LD, Evelo CT. A review of pathway-based analysis tools that visualize genetic variants. Front Genet. 2017; 8:174.

22. Cohen JA, Geradts J. Loss of RB and MTS1/CDKN2 (p16) expression in human sarcomas. Hum Pathol. 1997;28:893-8. 
23. El-Mokadem I, Fitzpatrick J, Bondad J, et al. Chromosome 9p deletion in clear cell renal cell carcinoma predicts recurrence and survival following surgery. Br J Cancer. 2014;111:1381-90.

24. Mascarello JT, Hirsch B, Kearney HMI, et al. A Working Group of the American College of Medical Genetics (ACMG) Laboratory Quality Assurance Committee. Section E9 of the American College of Medical Genetics technical standards and guidelines: Fluorescence in situ hybridization. Genet Med. 2011;13:667-75.

25. Assié G, Letouzé E, Fassnacht M, et al. Integrated genomic characterization of adrenocortical carcinoma. Nat Genet. 2014;46: 607-12.

26. Juhlin CC, Goh G, Healy JM, et al. Whole-exome sequencing characterizes the landscape of somatic mutations and copy number alterations in adrenocortical carcinoma. J Clin Endocrinol Metab. 2015;100:493-502.

27. De Martino MC, Al Ghuzlan A, Aubert S, et al. Molecular screening for a personalized treatment approach in advanced adrenocortical cancer. J Clin Endocrinol Metab. 2013;98:4080-8.

28. Cibulskis K, Lawrence MS, Carter SL, et al. Sensitive detection of somatic point mutations in impure and heterogeneous cancer samples. Nat Biotechnol. 2013;31:213-9.

29. De Krijger RR, Papathomas TG. Adrenocortical neoplasia: evolving concepts in tumorigenesis with an emphasis on adrenal cortical carcinoma variants. Virchows Arch. 2012;460:9-18.

30. Libè R, Groussin L, Tissier F, et al. Somatic TP53 mutations are relatively rare among adrenocortical cancers with the frequent 17 p13 loss of heterozygosity. Clin Cancer Res. 2007;13:844-50.
31. Erickson LA, Rivera M, Zhang J. Adrenocortical carcinoma: review and update. Adv Anat Pathol. 2014;21:151-9.

32. Ragazzon B, Libé R, Gaujoux S, et al. Transcriptome analysis reveals that $\mathrm{p} 53$ and \{beta\}-catenin alterations occur in a group of aggressive adrenocortical cancers. J Cancer Res. 2010;70:8276-81.

33. Ragazzon B, Assié G, Bertherat J. Transcriptome analysis of adrenocortical cancers: from molecular classification to the identification of new treatments. Endocr Relat Cancer. 2011;18:15-27.

34. Tissier F, Cavard C, Groussin L, et al. Mutations of beta-catenin in adrenocortical tumors: activation of the Wnt signaling pathway is a frequent event in both benign and malignant adrenocortical tumors. Cancer Res. 2005;65:7622-7.

35. Kim S, Xu X, Hecht A, Boyer TG. Mediator is a transducer of Wnt/beta-catenin signaling. J Biol Chem. 2006;281:14066-75.

36. Vignot S, Besse B, André F, Spano JP, Soria JC. Discrepancies between primary tumor and metastasis: a literature review on clinically established biomarkers. Crit Rev Oncol Hematol. 2012;84:301-13.

37. Vignot S, Frampton GM, Soria JC, et al. Next-generation sequencing reveals high concordance of recurrent somatic alterations between primary tumor and metastases from patients with non-small-cell lung cancer. J Clin Oncol. 2013;31:2167-72.

38. Walter MJ, Shen D, Ding L, et al. Clonal architecture of secondary acute myeloid leukemia. N Engl J Med. 2012;366:1090-8.

39. Vakiani E, Janakiraman M, Shen R, et al. Comparative genomic analysis of primary versus metastatic colorectal carcinomas. J Clin Oncol. 2012;30:2956-62. 